\title{
The Bruny Island Radio Spectrometer
}

\author{
W. C. Erickson \\ Physics Department, University of Tasmania, \\ GPO Box 252-21, Hobart, Tas. 7001, Australia \\ bill.erickson@utas.edu.au \\ Received 1997 March 20, accepted 1997 June 20
}

\begin{abstract}
A radio spectrometer has been built on Bruny Island, south of Hobart, for the study of solar bursts in the rarely observed frequency range from 3 to $20 \mathrm{MHz}$. This spectrometer is an adaptive device that employs digital techniques to avoid most of the strong terrestrial interference prevalent in this frequency range. The residual interference that cannot be avoided is excised during off-line processing. As a result, successful observations are made down to the minimum frequency that can propagate through the ionosphere to the antenna. This minimum frequency depends upon the zenith distance of the Sun and it is usually between 4 and $8 \mathrm{MHz}$.
\end{abstract}

Keywords: sun: radio radiation — instrumentation: miscellaneous

\section{Introduction}

There exists a number of scientific questions to be addressed by solar observations in the 2 to $20 \mathrm{MHz}$ frequency range. Energy releases, low in the solar atmosphere, produce coronal shock waves that generate coronal type II radio bursts. Ground-based observations have shown that these bursts usually die away in the 15 to $30 \mathrm{MHz}$ range. Similar bursts are observed from spacecraft at frequencies below $2 \mathrm{MHz}$ as interplanetary (IP) type II events and IP type II bursts are known to be generated by the IP shock waves associated with coronal mass ejections (CMEs). CMEs can have profound geophysical effects and it is of great importance to understand the physics of their origin and propagation. One key question is whether or not the IP shock waves are extensions of the coronal shock waves (Cane 1997). The most direct way to answer this question would be to observe a coronal type II burst that continued through the 20 to $2 \mathrm{MHz}$ range to become an IP type II event, or to show that this does not occur. However, for reasons discussed below, this frequency range is virtually unobserved. Other questions to be addressed through observations in the 2 to $20 \mathrm{MHz}$ range include the connection between shock associated (SA) events (Cane et al. 1981) observed in the IP medium and the herringbone structures that are observed in coronal type II bursts above $20 \mathrm{MHz}$. There appears to be a time correlation between these phenomena, but no direct connection has been made because of the frequency gap in the observations. SA events are of particular interest because they are well correlated with major proton events in the Earth's environment (Cane \& Stone 1984; Kahler, Cliver \& Cane 1986).

Radio observations from the surface of the Earth at frequencies below $20 \mathrm{MHz}$ are very difficult in the daytime because of high levels of terrestrial interference. Above $20 \mathrm{MHz}$, the interfering signals are normally far enough apart in frequency that solar bursts can be observed between them, but below this frequency interfering signals virtually fill the entire spectrum. This is because interfering signals in this frequency range propagate world-wide. Wideband, impulsive emissions from lightening strokes also propagate over long distances at frequencies below $20 \mathrm{MHz}$ and are another troublesome source of interference. Because of these problems, very few ground-based observations have been attempted.

Observations with space-borne systems are also uncommon in the 2 to $20 \mathrm{MHz}$ frequency range. Below the ionospheric critical frequency for vertical propagation $\left(f_{0} F_{2}\right)$ the ionosphere will normally shield spacecraft from Earth-generated interference. Daytime values of $f_{0} F_{2}$ are $3 \mathrm{MHz}$ or so, and above this frequency interference can break through the ionosphere to greatly disturb observations made from spacecraft located near the Earth. The problem is diminished by locating the spacecraft at a large distance from Earth, such as at the inner Lagrangian point, L1, but telemetry restrictions then limit the amount of spectral information that can be conveniently returned to Earth. For example, the WAVES experiment (Bougeret et al. 1995) on the WIND spacecraft, that is located at L1, observes up to $14 \mathrm{MHz}$ with limited spectral and temporal resolution.

Tasmania is known to have unusually low daytime ionospheric densities, which mitigates the problem of over-the-horizon interference from distant sources and allows solar bursts to propagate to the Earth's surface at quite low frequencies. On Bruny Island, an island off the southeast coast of Tasmania, locally generated interference is uncommon. Employing this 


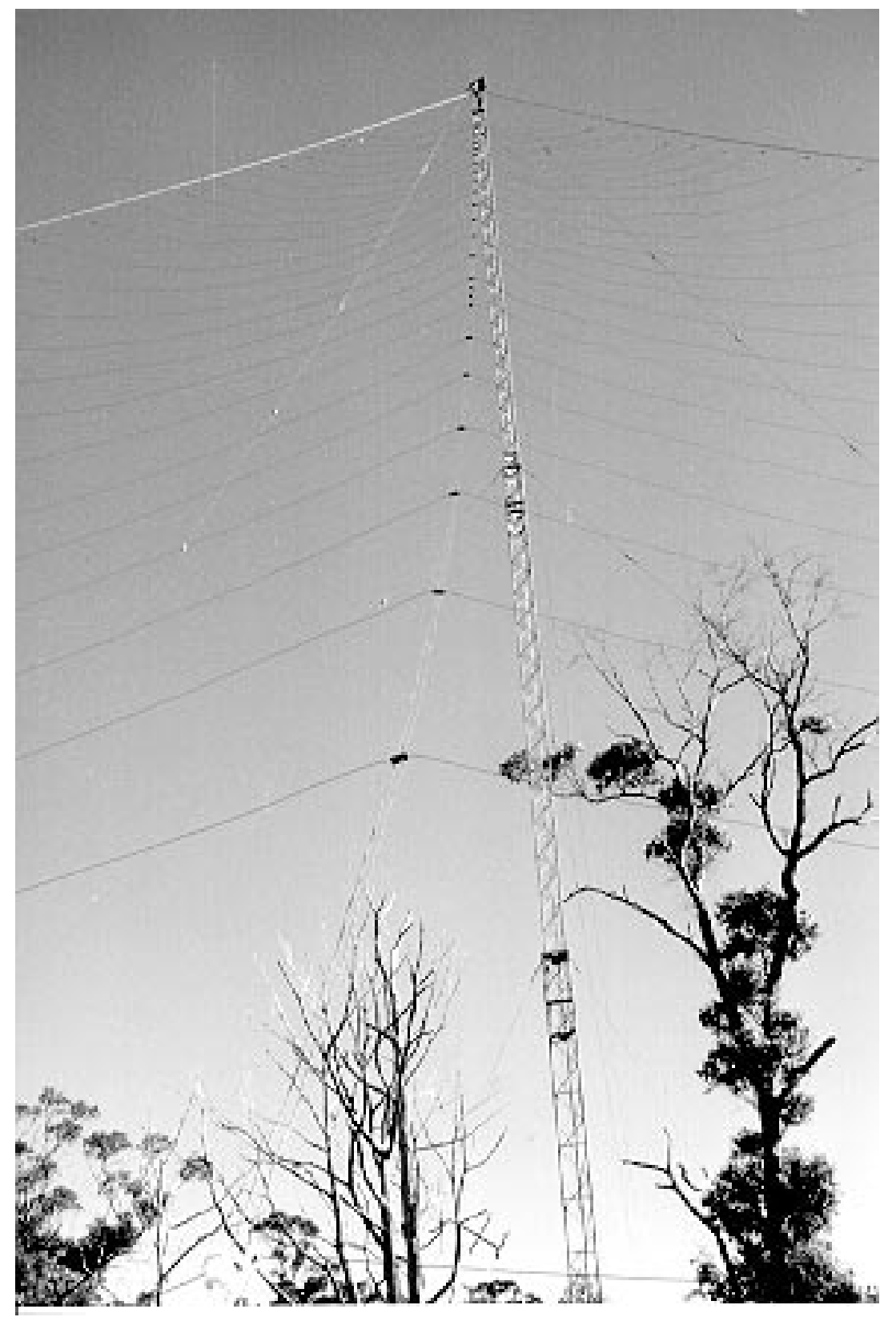

Figure 1-A photograph of the antenna.

good location from which to explore the possibilities for decametric solar observations, the Bruny Island Radio Spectrometer (BIRS) has been built. BIRS is an adaptive spectrometer designed to make use of modern digital techniques for the mitigation of terrestrial interference. The system is designed primarily for solar observations, but it is also usable for Jupiter observing and for monitoring any other transient decametric radio sources. At the present time, it is adjusted for swept-frequency observations in the 3 to $37 \mathrm{MHz}$ band. The upper portion of this band overlaps that covered by the Culgoora Radiospectrograph (Presage et al. 1994), which is at nearly the same longitude, making data comparison convenient. ${ }^{1}$ It has also been found useful to make detailed comparisons between BIRS and WAVES observations of the same bursts to delineate structure requiring high temporal and spectral resolution and to compare the flux density calibrations of the two instruments.

\section{The Antenna}

The geographic coordinates of the antenna are: Latitude $42 \cdot 370^{\circ} \mathrm{S}$, Longitude $147 \cdot 218^{\circ} \mathrm{E}$. As shown in Figure 1, the antenna is a 23 element log-periodic structure fixed in position and pointed to the

\footnotetext{
${ }^{1}$ The original Culgoora spectrograph operated down to $8 \mathrm{MHz}$ but did not produce very much useful data in the lower part of its frequency range. When the spectrograph was rebuilt in 1992 the lower limit of its operating range was changed to $18 \mathrm{MHz}$.
} 
celestial equator near the meridian. Its response pattern is broad enough to cover the whole ecliptic and to have reasonable hour angle coverage. The antenna's directivity is low, about $6 \mathrm{db}$ /isotropic; its horizontal E-plane pattern is about $90^{\circ}$ wide and its vertical $\mathrm{H}$-plane pattern is more than $100^{\circ}$ wide. The antenna has an opening angle of $90^{\circ}$ at its apex, and a scale factor of $0 \cdot 89$. The longest dipole is $50.0 \mathrm{~m}$ and the largest spacing between dipoles is $2 \cdot 75 \mathrm{~m}$. These values decrease geometrically by the scale factor to the shortest, $3.05 \mathrm{~m}$, dipole. The height of the tower which supports the apex of the antenna is $21.4 \mathrm{~m}$. The geometrical bandwidth of the structure is 3 to $45 \mathrm{MHz}$ but, since the radiating region spans an appreciable length of the structure at any single frequency, the RF bandwidth of the system is $3 \cdot 3$ to $40.9 \mathrm{MHz}$. Interference effects between the direct ray and the ray reflected from the ground, i.e. Lloyd's mirror effects, are observed, but the reception of the reflected ray is diminished by 5 to $20 \mathrm{db}$ because of the antenna's directivity. These effects are calculated and partially removed in the data processing. The instrument is normally operated from $22^{h} 00^{m}$ UT to $06^{h} 30^{m}$ UT each day, although the directivity of the fixed antenna limits the observing period to about 6 hours with good sensitivity.

\section{Preamplification}

A preamplifier, located at the base of the tower, employs a small, high dynamic range, monolithic amplifier with a noise figure of $3 \cdot 6 \mathrm{db}$. This wideband $(1-1000 \mathrm{MHz})$ device is protected with filters to restrict its bandwidth to 3 to $45 \mathrm{MHz}$. Its gain is kept low $(13 \mathrm{db})$ to avoid any possibility of overloading on strong interference. However, the gain is sufficient to overcome line losses between the antenna and the laboratory.

Before preamplification the antenna signals pass through a $10 \mathrm{db}$ directional coupler for injection of calibrations. After preamplification the signals are transmitted to the laboratory through $125 \mathrm{~m}$ of buried coaxial cable. In the laboratory a slope filter attenuates the signals below $18 \mathrm{MHz}$ by $20 \mathrm{db}$. This avoids any possibility of overloading by strong low frequency interference while the noise generated by the Galactic background radiation still dominates the system noise. After $25 \mathrm{db}$ of amplification in a high power amplifier, the signals are presented to a spectrum analyser.

\section{Spectrum Analysis}

The signal spectrum is analysed with a commercial spectrum analyser that is controlled by a $386 \mathrm{PC}$. The analyser operates from 3 to $37 \mathrm{MHz}$ with an instantaneous bandwidth of $10 \mathrm{kHz}$. The spectrum is sampled at 128 points every 3 seconds. The detected output signal from the analyser is linearly integrated at each frequency step for about $20 \mathrm{~ms}$, then A/D converted and sent to the PC that controls the system and acquires the data. Every 30 minutes the system makes a full scan of the spectrum, from 3 to $37 \mathrm{MHz}$, with $10 \mathrm{kHz}$ resolution. It then finds any 'holes' between interference transmissions in each $265 \cdot 6 \mathrm{kHz}$-wide section of the spectrum and, for the next 30 minutes, it observes only in these 128 'holes'. After 30 minutes the system re-adapts itself to the constantly changing interference environment. The data are displayed in frequency-time coordinates on a monitor with signal intensity on the colour axis. The data are also written to disk every 30 minutes and, at the end of each day, they are compressed and automatically transferred to tape for off-line processing and long-term storage.

Without these adaptive procedures to avoid most of the interference, observations below $20 \mathrm{MHz}$ would be practically impossible and, in spite of these procedures, high interference levels from over-thehorizon sources remain. In addition to man-made interference, wide-band spikes caused by lightening, both local and distant, are common. An off-line filtering program has been developed which reduces the response of the system to steady narrow-band signals and to instantaneous wide-band spikes. As illustrated in Figure 2, the output of this filtering program produces records of solar emissions that are virtually interference-free.

\section{Data Description and Calibration}

The system began operation in mid-1994. Since the level of solar activity has been low, only about a dozen type II bursts have been observed thus far. All of them have stopped within the observed frequency range. Hundreds of type III bursts have been observed from $37 \mathrm{MHz}$ down to the ionospheric cut-off. This cut-off is usually 4 to $8 \mathrm{MHz}$ but the cut-off frequency depends strongly upon the zenith distance of the Sun. The $3 \mathrm{MHz}$ limit to the observed frequency range ensures that any emission that can possibly get through the ionosphere in daytime will be observed. When the cut-off has been below $3 \mathrm{MHz}$ late at night, some bursts, believed to be from Jupiter, have been observed down to the $3 \mathrm{MHz}$ limit of observing range.

The response of the receiving system has been carefully calibrated with a thermionic diode and the antenna gain has been estimated theoretically. The polar galactic background spectrum has been observed late at night and this observed galactic spectrum agrees well with calibrated, fixed-frequency measurements made by other observers. Appreciable ionospheric absorption occurs below $15 \mathrm{MHz}$ in daytime. In order to estimate and correct for this effect, during data analysis an apparent Galactic 


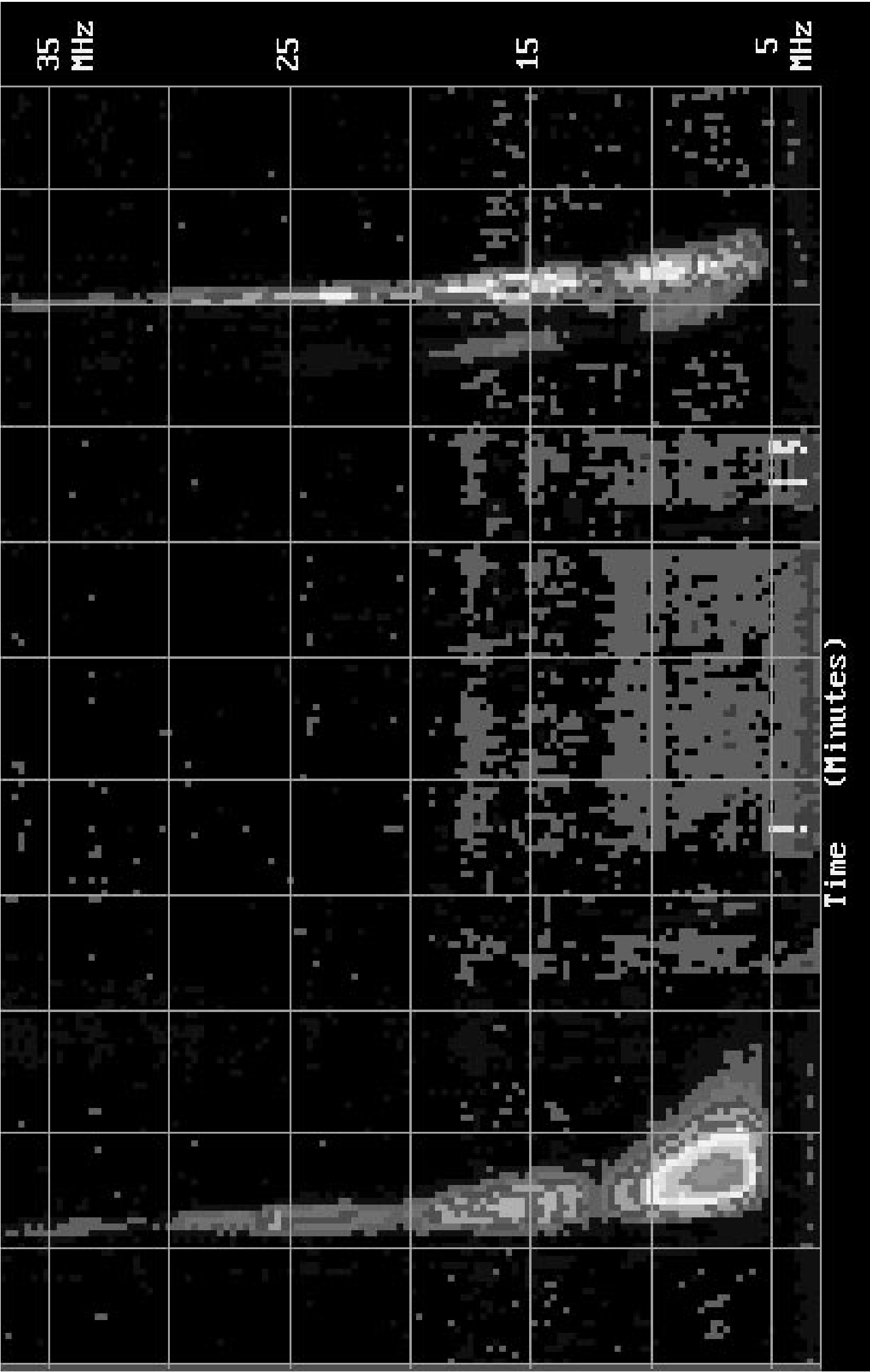

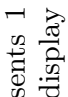

苛.

응

它志

矛过

ن

要,

:

蛋

B. 2 ?

$\vec{i}$

䒕

出

$\exists$ 잉

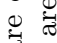

窟

$\mp$ 范

过

उ

灵

is 50

요

救 
background spectrum is determined for a time adjacent to the solar burst. This apparent spectrum is then compared with an archived, absorption-free spectrum that was previously observed at the same Right Ascension late at night, and the amount of daytime absorption is then estimated.

\section{Summary}

A radio spectrometer has been designed and constructed for ground-based solar observations in the poorly covered frequency range below $20 \mathrm{MHz}$. Techniques have been developed to avoid and excise the abundant interfering signals prevalent in this frequency range. The system scans the radio spectrum from 3 to $37 \mathrm{MHz}$ and provides nearly interferencefree records of solar radio bursts with 3 second time resolution, $268 \mathrm{kHz}$ frequency resolution, and a well-calibrated flux density scale. The instrument is normally operated from $22^{h} 00^{m}$ UT to $06^{h} 30^{m} \mathrm{UT}$ each day.

\section{Acknowledgments}

The author wishes to acknowledge the enthusiastic support and assistance given to this project by his wife, Hilary V. Cane. Various items of equipment, including a spectrum analyser, were donated by the Hewlett-Packard Corporation through the cooperation of Mr John Hubbard.

Bougeret, J.-L., et al. 1995, Space Sci. Rev., 71, 231

Cane, H. V. 1997, Coronal Mass Ejections: Causes and Consequences, ed. N. Crooker et al. (AGU Monograph Series)

Cane, H. V., \& Stone, R. G. 1984, ApJ, 282, 339

Cane, H. V., Stone, R. G., Fainberg, J., Stewart, R. T., Steinberg, J.-L., \& Hoang, S. 1981, Geophys. Res. Lett., 8,1285

Kahler, S. W., Cliver, E. W., \& Cane, H. V. 1986, Adv. Space Res., 6, 319

Prestage, N. P., Luckhurst, R. G., Paterson, B. R., Bevins, C. S., \& Yuile, C. G. 1994, Solar Phys., 150, 393 